\title{
Short Courses for Scanning Electron Microscopy and X-ray Microanalysis
}

\author{
Charles E. Lyman
}

Department of Materials Science and Engineering, Lehigh University, 5 East Packer Avenue, Bethlehem, PA 18015

Scanning electron microscopy (SEM) combined with energy-dispersive x-ray spectrometry (EDS) and electron backscatter diffraction (EBSD) comprise the research tool of choice for many imaging and analysis tasks in biology, geology, materials science, and nanotechnology. This instrument combination is so powerful that many projects can benefit from just a few images and a couple $\mathrm{x}$-ray spectra from regions within those images. These flexible instruments also, however, can solve many types of difficult problems at the nanoscale if the full range operation conditions can be utilized. Unfortunately, many people using SEM instrumentation today have not had an academic SEM course or even an SEM manufacturer's on-site or demolab course. Yet sooner or later most SEM operators will be asked to provide interpretation of their data as well as suggestions as to how to get more detailed information. A one-week short course can provide the background to help the SEM user understand the interpretation of images, spectra, and diffraction data.

Short courses should provide an enriching experience for all participants, whether they are expert SEM operators or novices. This experience should take place in a fun-filled week with good food and ample time for social interactions. Successful SEM short courses share at least some of the following characteristics: (1) several experienced lecturers and lab instructors, (2) textbooks written by the lecturers, (3) clear links between the lecture topics and the illustrative laboratory exercises, (4) an atmosphere that encourages student questions and discussion with the lecturers, and (5) continuous improvement in which the organizers heed the comments of the participants.

At the Lehigh Microscopy School [1], each of the six courses is presented by several experts. For the main SEM course, Scanning Electron Microscopy and X-ray Microanalysis, there are 18 lecturers and lab instructors not counting instrument specialists sent by the companies that provide equipment for the course. This large number of lecturers provides: (a) several levels of depth, useful for beginners and experienced operators alike, (b) a wealth of examples drawn from many application areas, (c) expertise with accessories and specialized equipment, (d) total immersion in microscopy techniques during lectures and lab sessions, and (e) opportunities to ask questions in relaxed settings.

A typical SEM topic is presented in four steps. First, the topic is introduced in the lecture session accompanied by a full set of lecture notes (Figure 1). Second, the same topic is presented live on a microscope to demonstrate the principles discussed in lecture (Figure 2). A laboratory workbook guides students through the setups and operations so that the same experiments can be reproduced on the instrument in their home laboratory. The number of students in each lab group is small so that everyone can see, hear, and ask questions. Third, some of the laboratory exercises utilize the Lehigh Microscopy School software provided to each participant. Finally, each student receives an authoritative textbook written by the lecturers [2]. Figure 3 shows how the lecture presentation, laboratory session, and laboratory workbook 
combine to present the topic of qualitative x-ray spectrometry. These same pedagogical methods are used in all six courses of the Lehigh Microscopy School: Scanning Electron Microscopy and X-ray Microanalysis, Introduction to SEM and EDS, Focused Ion Beam Instrumentation and Applications, Problem Solving with SEM/EDS/EBSD, Quantitative X-ray Microanalysis, and Scanning Transmission Electron Microscopy [1].

\section{References}

[1] http://www.lehigh.edu/microscopy/school/index.html.

[2] J.I. Goldstein et al., Scanning Electron Microscopy and X-ray Microanalysis, Springer, 2003.

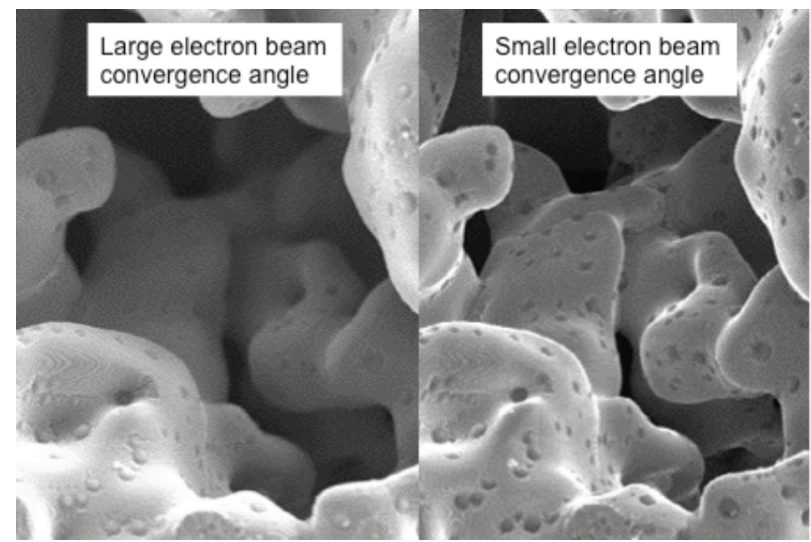

Fig. 1. Lecture example of SEM depth of field.

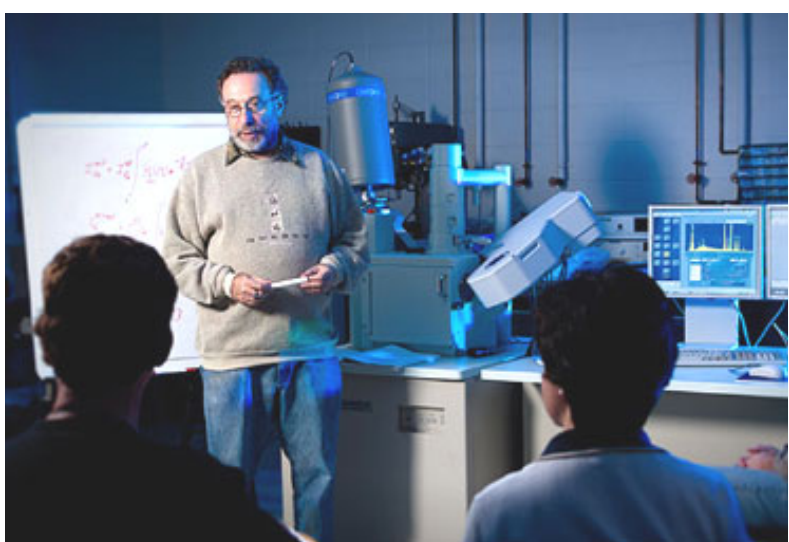

Fig. 2. Lab session on x-ray microanalysis.

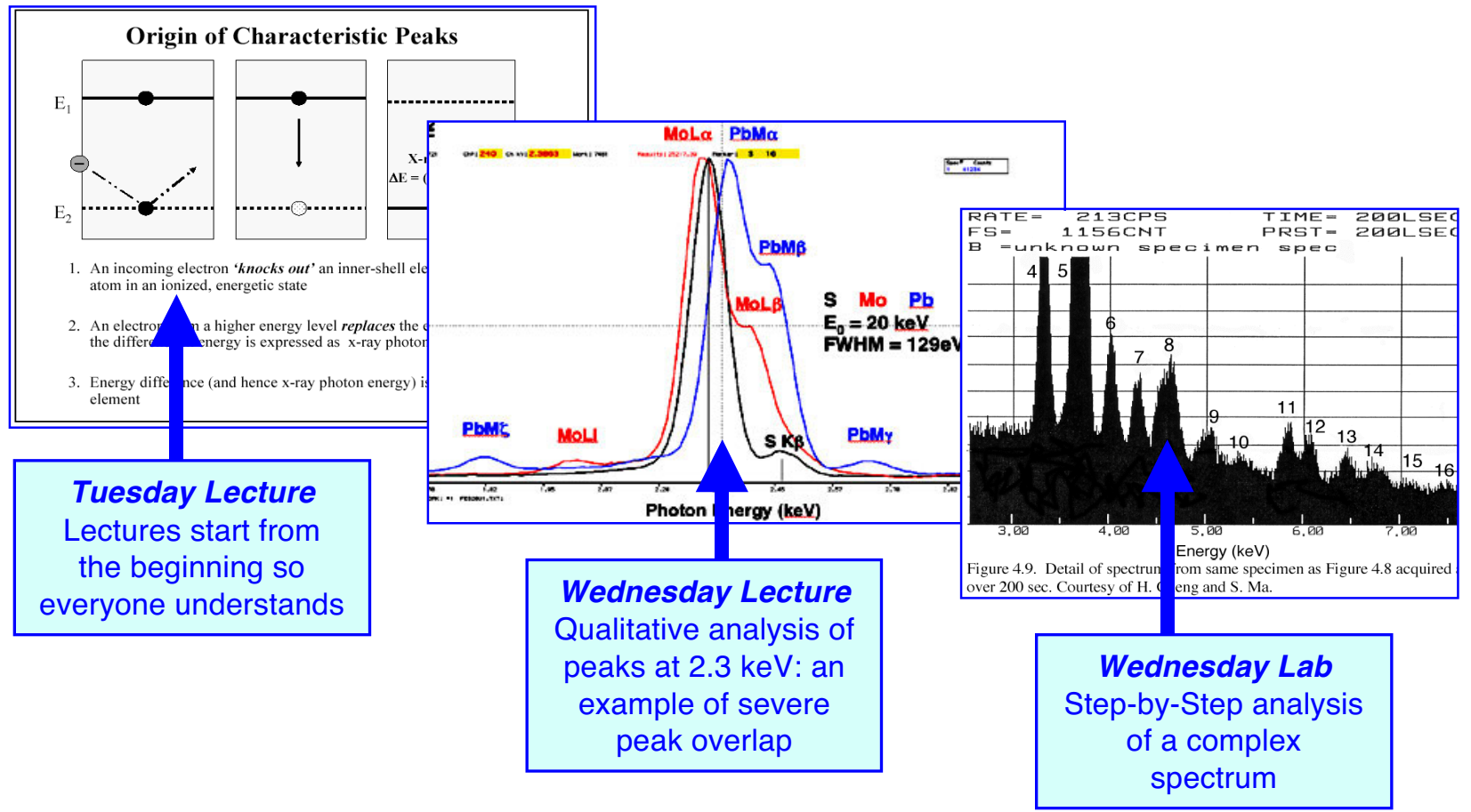

Fig. 3. Pedagogy for teaching qualitative $x$-ray microanalysis at a short course. Understanding the details of $\mathrm{x}$-ray analysis is important for correct interpretation of data. 